\section{NogerTsien $1952-2016$}

David Kleinfeld
Turn back the clock by forty years. Disco was fading and the Ramones were about to inject life back into a moribund rock music scene. And neuroscience was operating in a parallel universe. The electrode was king. Microscopy was for histology. Functional imaging was outside our lexicon. A few brave souls were using natural optically active proteins to measure cell signaling. This included the luminescent calcium sensor Aequorin, ironically isolated from the same jellyfish endowed with green fluorescent protein. A decade later the technological front for measuring neuronal activity was rapidly morphing from electrodes and amplifiers to lenses and CCD cameras. Fura-2, an easily loaded ratiometric fluorescent indicator, had burst on the scene. By the late 1980s the yearly meeting of the Biophysical Society was transformed from a staid affair into a Technicolor wonderland of posters on calcium measured in nearly every kind of cell. The electrode now had a partner, ushered in by Roger Tsien, who even as a precocious upperclassman realized that synthetic chemistry could provide a new avenue for probing the function of cells.

Roger, our brilliant and visionary colleague, died last month. He had fought back from the ravages of a stroke some three-and-a-half years before then. A tough guy and a dedicated scientist to the end, he was completing old projects and starting on new ones. It was as if a thirst for knowledge was an elixir that helped to keep him going.

The University of California, San Diego (UCSD) is nothing if not a collaborative environment, and I had the good fortune to join Roger on a few projects. Roger was closely involved in the planning and interpretation of experiments and, despite jokes about the "minimal publishable unit," he tirelessly supported thoroughness. He was efficient with his time and could be quite abrupt. Yet, away from UCSD, on a trip somewhere with emotional defenses weakened by jet lag, Roger would open up and talk. About growing up in New York and working on projects with his dad. About clearing a seeming infinity of rocks from the yard of his family's then new home in New Jersey. About his days at Cambridge. And, touchingly, about meeting Wendy, his wife, at a Christmas party. But the proverbial 'walk in the woods' generally avoided gossip and eventually yielded to discussions on science.

Roger was in awe of biology. Yet what Roger liked best, and in fact did better than any of us, was to create tools. He used his knowledge of synthetic chemistry to create new ways of probing the locations and concentrations of ions and molecules that cells used to signal. He had an uncanny ability to grasp fundamental relationships such as the trade-off between specificity versus speed and to overcome practical issues such as the difficulty of loading dye into a cell.

As a postdoc, Roger exploited three essential insights into the design of molecular probes, and these drive the field to this day. First, he recognized the importance of fluorescence, which affords greater sensitivity than transmittance for measuring changes in optical activity. Next, he saw the necessity of developing a proper chemical framework for probes.

David Kleinfeld is in the Department of Physics (primary) and Section of Neurobiology, University of California, San Diego, La Jolla, California, USA. e-mail: dk@physics.ucsd.edu
This, in particular, led to creation of BAPTA, a highly conjugated, rapidly equilibrating calcium buffer that is still the basis of modern organic indicators. Finally, motivated by the prodrug method, he linked the indicator with a lipophilic molecule so that the dye was trapped in a cell after the link was metabolized. Fura-2 AM was the second-generation product of this approach. Its use led to spatially resolved optical measurements of the intracellular concentration of $\mathrm{Ca}^{2+}$, a key second messenger, across a wide range of neurons and other cell types.

Having brought neuroscience into the age of light through the optical detection of calcium, Roger shifted his focus to the detection of biomolecules. The first stop was cyclic adenosine monophosphate (cAMP), a target that was motivated by a desire to understand the trafficking of protein kinase A (PKA). Interestingly, Roger transformed this from a question about PKA to a project to form a new indicator based on the cAMP-mediated dissociation of the subunits of PKA. Through savvy synthesis, Roger labeled the subunits with different fluorophores and made use of fluorescence resonant energy transfer (FRET) to optically detect the state of dissociation versus coalescence. In an early application, Roger and his colleagues determined the flux of cAMP into the cell nucleus that was required to initiate long-term synaptic changes.

While the prodrug trick enabled loading of exogenous dyes and indicators without the need for an electrode, Roger looked for a means to intrinsically express dyes in cells. He noted that "... I typed 'fluorescent protein' into PubMed, was reminded of Osamu Shimomura's early work on green fluorescent protein [GFP], and then learned that GFP had been cloned by a fellow named Douglas Prasher." Doug, rapidly depleting his grant funds, bet on a long shot and hoped fluorescence would come from expressing only a piece of the protein. This failed. In an act of academic desperation, Doug graciously sent his clone to Roger, as well as to Martin Chalfie. Marty was able to express wild-type GFP in the worm Caenorhabditis elegans. Roger, delayed by seeking expression in yeast and belatedly switching to bacteria, did an end run to stay in the game. He used his prodigious skills in chemistry and newfound skills in molecular biology to modify the protein, which considerably simplified the absorption spectrum and led to the S65T variant; with changes to codon usage, this became the now ubiquitous enhanced GFP (eGFP). This protein served as a template for Roger and his colleagues to produce blue, cyan (eCFP) and yellow (eYFP) variants. Red fluorescent protein (RFP) was soon discovered by Sergey Lukyanov in corals. Roger's laboratory transformed RFP from a tetramer to a monomer and then pushed forward to form an array of proteins that fluoresce at long wavelengths. In less than one decade the field of genetically expressible indicators went from a few natural proteins that required external cofactors to an artist's palette of readily expressible chimeric proteins, whose emitted light ranged from deep purple to far red.

A next step for Roger was to modify fluorescent proteins for use as genetically expressible indicators of calcium. The first idea was to identify a protein, or protein complex, that changes shape upon binding a ligand, $\mathrm{Ca}^{2+}$ in this case, and then use this change to alter the distance between a pair of fluorescent proteins that interact through FRET. This approach led to the first family of genetically expressible sensors for 


\section{O B IT UARY}

calcium, the cameleons, which were constructed as a chain of three parts: eYFP, a sensor derived from peptides that coalesce in the presence of $\mathrm{Ca}^{2+}$, and eCFP. Cameleon worked well in C. elegans but had limited success in mammalian neurons. Nonetheless, it was a pioneering step toward genetically expressible indicators.

While the currency of the nervous system is the action potential, present day voltage indicators lack sufficient sensitivity in vivo. Calcium transients, the long-lived ghost of spikes, serve as a surrogate. Currently, the brightest and most sensitive genetically expressible $\mathrm{Ca}^{2+}$ indicators are the single-fluorophore GCaMPs. The development of these probes was critically dependent on a modification of eGFP that resulted from Roger's insight into protein chemistry. His laboratory showed that the amino and carboxy termini of eGFP could be relocated to positions where twisting the termini will alter the fluorescence. This so-called cyclically permuted eGFP forms the core of GCaMP, in which the twist is supplied by the $\mathrm{Ca}^{2+}$-mediated coalescence of amino- and carboxyterminal protein tags. Targeted expression is then used to label all neurons, or all neurons of a common genetic driver, with this robust $\mathrm{Ca}^{2+}$ indicator. Neuroscientists can now record from many neurons in a brain, even all neurons in a small brain.

How do we summarize Roger's impact? Winfried Denk, another pioneer of neurotechnology, puts it best. "Roger was, or should have been, the hero of all of us who develop new methods. He always asked himself, what could he create that would allow others to answer questions that they couldn't yet?" We mourn the loss of an irreplaceable colleague. Yet we honor Roger by understanding the logic of his insights and by thinking, and pressing forward, as boldly as we dare.

\section{ACKNOWLEDGMENTS}

I extend special thanks to S.R. Adams, who worked closely with Roger since Adams' days as a postdoc, and M.H. Ellisman, Roger's long-term collaborator at UCSD, for extended conversations. I further thank L.B. Cohen, W. Denk, B. Friedman, Y. Jin, W.N. Ross and J.W. Wang for discussions. My summary of Roger's academic life is biased toward his tools to measure cell signaling in the context of neuroscience and is at best a vignette of a life's work. 